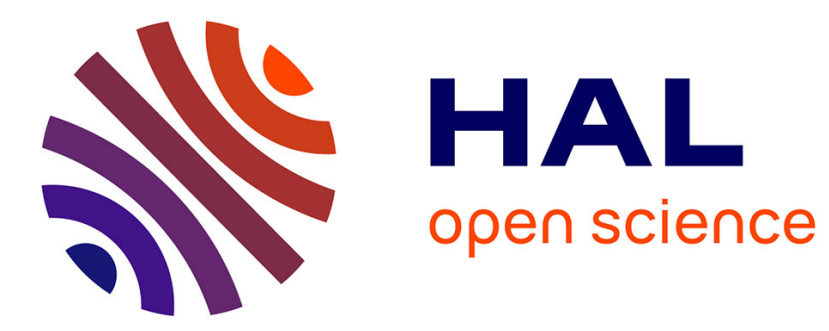

\title{
Trajectory estimation for a hybrid rocket
}

Pierre-Jean Bristeau, Nicolas Petit

\section{To cite this version:}

Pierre-Jean Bristeau, Nicolas Petit. Trajectory estimation for a hybrid rocket. 2009 AIAA Guidance, Navigation, and Control Conference, Aug 2009, Chicago, United States. pp.1-9. hal-00525799

\section{HAL Id: hal-00525799 \\ https://hal-mines-paristech.archives-ouvertes.fr/hal-00525799}

Submitted on 12 Oct 2010

HAL is a multi-disciplinary open access archive for the deposit and dissemination of scientific research documents, whether they are published or not. The documents may come from teaching and research institutions in France or abroad, or from public or private research centers.
L'archive ouverte pluridisciplinaire $\mathbf{H A L}$, est destinée au dépôt et à la diffusion de documents scientifiques de niveau recherche, publiés ou non, émanant des établissements d'enseignement et de recherche français ou étrangers, des laboratoires publics ou privés. 


\title{
Trajectory estimation for a hybrid rocket
}

\author{
Pierre-Jean Bristeau* and Nicolas Petit ${ }^{\dagger}$ \\ Centre Automatique et Systèmes, Unité Mathématiques et Systèmes, MINES ParisTech, Paris, FRANCE
}

\begin{abstract}
This paper presents a research work to develop a navigation technique for a class of nano-micro space rocket. Onboard MEMS inertial sensors are used in view of post-flight trajectory estimation. Combined with synchronized measurements of the combustion engine which is of hybrid type and uses $\mathrm{N}_{2} \mathrm{O}$ and $\mathrm{PE}$, we show how the relative redundancy of the data can be used to calibrate a thrust model. Experimental results are presented and stress the numerous difficulties needed to be resolved. Quantitative results are provided along with estimates of the obtained accuracy.
\end{abstract}

\section{Introduction}

Nano launchers, i.e. launcher capable of putting a typical payload of $10 \mathrm{~kg}$ at an altitude of $250 \mathrm{~km}$, have been the subject of a substantial research effort over the last decades. In this field, hybrid rockets have attracted much attention ${ }^{1}$ because of their low cost, good performance and safety record. A main reason is the emergence of an interest of end-users for short terms space missions where tiny satellites are to be used for a short period (a few days) in a specific geographic region. Prime examples are UNO related missions (to monitor sudden disasters such as flooding or earthquakes), or military operations requiring specific observation and/or communication capabilities. Another interest of such systems is their relatively low cost, due to the possibility of small series mass production, to attract new potential users such as metrological and microgravity researchers. ${ }^{2}$

In this context, several projects have been under development. In particular, we present here a study being part of the PERSEUS project ${ }^{3}$ conducted by Centre National d'Etudes Spatiales (CNES). These systems must rely on lowcost technologies. This is also true for all the included subsystems ranging from the propulsion system, the mechanical structure, and very importantly the navigation system which is the subject of the present paper.

Space launcher navigation systems usually incorporate high quality inertial sensors (accelerometers, gyroscopes) ranging in the upper class of the sensors found in aerospaces systems (aircraft, missiles). ${ }^{4}$ This requirement is due to the lack of possible reconciliation of data with extraneous information (GPS, or pilot instructions), the total flight time which can be as large as 40 minutes, and the quality requirements of navigation signal for the stabilizing closedloop controllers. Due to their costs and weights, such high quality sensors must be discarded for nano/micro rockets. Fortunately, the specifications of these rockets are also different. In particular, the flight time is much lower, which enables to use possibly biased and noisy signals.

Therefore, it seems reasonable to use MEMS inertial sensors on-board such a rocket and to integrate the equations of motion to obtain an estimate of the trajectory. In practice, only a real experiment can support this fact. This is the purpose of the presented research.

We consider a very small rocket ( $2.5 \mathrm{~m}$ high) equipped with MEMS inertial sensors. This rocket is of hybrid type. It uses $\mathrm{N}_{2} \mathrm{O}$ and polyethylene (PE) to generate a thrust of approximately $1000 \mathrm{~N}$. The ascent trajectory lasts less than 9 sec. This rocket, named FH02, belongs to the smallest class of rockets considered in the PERSEUS project. Soon, further developments will include a second class of rockets capable of reaching an altitude of $15 \mathrm{~km}$, which will be followed by a third class targeting the altitude of $100 \mathrm{~km}$.

Following the philosophy of ${ }^{5}$ for hardware selection, off-the-shelf components are used to build the navigation system. An MTi® inertial measurement unit (IMU) from XSens (incorporating a 3-axis accelerometer, 3 gyroscopes, and 3 magnetometers) is embedded in the rocket along with a data logger. The data logger also stores measurements from the combustion chamber. In details, high frequency signals from pressures sensors in the reservoir and in the combustion chamber are stored along with reservoir temperature sensors.

\footnotetext{
*PhD candidate

${ }^{\dagger}$ Professor, AIAA Member

*This work was partially supported by CNES and by SYSNAV
} 
The outcome of this study are as follows. First, it is demonstrated that the ascent trajectory of such a hybrid rocket can be obtained with reasonable accuracy. Secondly, we show that a combustion model expressing the thrust generated by the hybrid reduction/oxidation chemical reaction can be fitted from in-flight data. This is of particular interest since the calibration of such a model is very difficult to achieve on the ground, i.e. on a test bench simulating in-flight conditions.

The paper is organized as follows. Section II presents the rocket under consideration and its model which consists of a 6 degrees of freedom (DOF) dynamics, and a thrust model. Aerodynamics effects are also discussed. Section III exposes the trajectory estimation problem and the obtained results. Finally, conclusions and future directions are given in Section IV.

\section{Hybrid rocket under consideration}

\section{A. Description}

The hybrid rocket under consideration is as follows. It consists of three main parts: the engine in the lower part of the body, the parachute box in the middle zone, and the electronic devices in the higher part. The mass distribution and the induced location of the center of gravity above the center of aerodynamics forces guarantees aerodynamic stability.

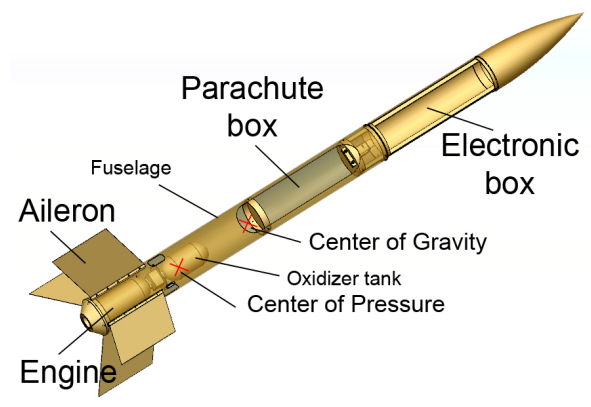

\begin{tabular}{|c|c|}
\hline Height & $2.5 \mathrm{~m}$ \\
\hline Diameter & $150 \mathrm{~mm}$ \\
\hline acceleration level & $100 \mathrm{~m} \cdot \mathrm{s}^{-2}$ \\
\hline al max. altitude & $460 \mathrm{~m}$ \\
\hline
\end{tabular}

Figure 1. Hybrid rocket components and specifications.

\section{B. Model}

We now detail a model for the hybrid rocket under consideration here. It is considered as a rigid body with 6 D-O-F subjected to a thrust generated by the engine. A nomenclature is given below.

\section{Nomenclature}

$M \quad$ Mass, $\mathrm{kg}$

$J \quad$ Moment of inertia tensor, kg.m²

$V \quad$ Velocity vector relative to an inertial frame, $\mathrm{m} . \mathrm{s}^{-1}$

$\Omega \quad$ Angular velocity vector of the body frame relative to an inertial frame, $\mathrm{s}^{-1}$

$\sum F$ sum of external force vectors, $\mathrm{N}$

$\sum M$ sum of external moment vectors, N.m

$\frac{d}{d t} \quad$ derivation operator compared to an inertial frame

$\frac{\partial}{\partial t} \quad$ derivation operator compared to the body frame

\section{Variable mass rigid body}

Based on Kane's formalism and volume integrals, ${ }^{6}$ under the assumption of axi-symmetric and/or negligible displacements of matter inside the rocket, the dynamics of a variable mass rigid body is given by the following equations, 
where $V$ is the speed relative to an inertial frame of a fixed point of the rocket and CG is the vector distance of this point with respect to the center of gravity

$$
\begin{aligned}
& M\left(\frac{d V}{d t}+\dot{\Omega}_{\wedge} C G+\Omega_{\wedge}\left(\Omega_{\wedge} C G\right)\right)=\sum F+F_{t}+F_{c} \\
& J \frac{d \Omega}{d t}+\frac{\partial J}{\partial t} \Omega=\sum M+M_{d}
\end{aligned}
$$

The mass loss induces the force $F_{t}$ which is the thrust, the force $F_{c}$ which is the Coriolis acceleration and the moment $M_{d}$ which is a damping moment.

In the case considered here, the maximum loss of max is relatively small compared to the total mass of the rocket. Therefore, the displacement of the center of gravity is small and slow, and one can neglect some terms compared to the thrust. Under this assumption, the dynamics write

$$
\begin{aligned}
& M\left(\frac{\partial V}{\partial t}+\Omega_{\wedge} V\right)=\sum F+F_{t}+F_{c} \\
& J \frac{\partial \Omega}{\partial t}+\Omega_{\wedge} J \Omega+\frac{\partial J}{\partial t} \Omega=\sum M+M_{d}
\end{aligned}
$$

Among the external forces are the gravity, the pressure force which is applied at the nozzle exit, the aerodynamic forces which are applied at the center of pressure, and the force exerted by the parachute when it is deployed. The various lever arms generate external torques. To determine the moment $M_{d}$ and the effect due to the inertia variation requires the knowledge of the mass distribution during the combustion.

\section{Thrust model: hybrid propulsion}

The considered rocket has a classic multi-port hybrid engine ${ }^{78}$. It uses an oxidizer $\mathrm{N}_{2} \mathrm{O}$ which is stored in liquid form in a tank and solid fuel (PE). The combustion is self-pressurized: when the compressed oxidizer tank is opened, the $\mathrm{N}_{2} \mathrm{O}$ flows to the combustion chamber through the injector. In gaseous phase, it reacts with the fuel which constitutes the chamber envelope. Then, the thermo-chemical reaction generates mass ejection at high speed.

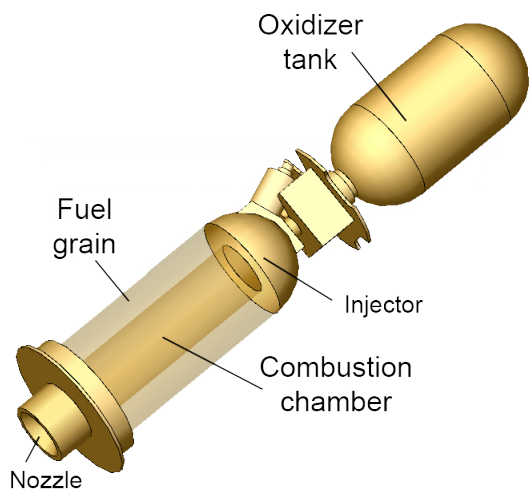

Figure 2. Hybrid engine.

For navigation purposes, the thrust model is important to predict the thrust level but also to determine the massflow rate and thus the mass distribution, i.e. the position of the center of gravity and the inertia tensor. To monitor the thrust generation, the hybrid engine is equipped with three particular sensors: the tank temperature $T_{r}$, the pressure $P_{r}$ inside the oxidizer tank, and the pressure $P_{c h}$ in the combustion chamber are measured at high frequency. In details, two phases can be distinguished during the combustion: the first phase when the oxidizer gets out the reservoir in the liquid phase, and the second phase, called "tail of combustion", during which the pressure in the oxidizer tank is so low that the $\mathrm{N}_{2} \mathrm{O}$ is already under gaseous phase in the tank before it goes to the combustion chamber without any phase change. During the first phase of combustion, pressure oscillations around $200 \mathrm{~Hz}$ can be observed.

Under the hypothesis of steady operations, in the oxidizer tank, one can formulate an equation of the oxidizer mass-flow rate $\dot{m}_{o}$ which represents the oxidizer mass loss and the engine regime change. In details, a liquid and a 
gaseous phase are modeled in the tank. The density $\rho_{o}$ depends on the phase under which the oxidizer comes out the tank.

$$
\dot{m}_{o}=k \sqrt{2 \rho_{o}\left(P_{r}-P_{c h}\right)}
$$

Knowing the oxidizer mass-flow rate, one can compute the oxidizer mass flux $G_{o}$ and, then, the regression rate $\dot{r}$ of the fuel grain

$$
\begin{aligned}
& G_{o}=\frac{\dot{m}_{o}}{N \pi r^{2}} \\
& \dot{r}=a G_{o}^{n}
\end{aligned}
$$

The model parameters $a$ and $n$ can be determined so that the regression rate verifies the initial and final conditions. This is how the fuel mass flux and the oxidizer-to-fuel ratio can be computed. Further, a thermo-chemical model, taking the form of look-up tables, gives the characteristic exhaust velocity $c *$ and the specific impulse $I_{s p}$ as functions of the oxidizer-to-fuel ratio and of the chamber pressure.

It can be checked that the ejected mass-flow rate is consistent with the total mass loss

$$
\dot{m}_{e}=A_{t} g / c *
$$

where $A_{t}$ is the throat cross-sectionnal area and $\mathrm{g}$ is the gravity.

Finally, the theoretical thrust force is expressed as an indirect function of the measurements available on the engine. This takes the following form

$$
F_{T}=\dot{m}_{e} I_{s p} g \triangleq f\left(T_{r}, P_{r}, P_{c h}\right)
$$

\section{Aerodynamics}

For this kind of small experimental rockets, the aerodynamic modeling is not essential. On one hand, drag effects are negligible compared to the thrust force since the airspeed does not reach important values in a so short acceleration time (less than $2 \mathrm{sec}$ ). On the other hand, transverse effects like incidence oscillations (short period mode) are difficult to identify because of large uncertainties on aerodynamic parameters (like the lift coefficient) and mechanical parameters (principally the inertia tensor). Fortunately, they are well visible on the gyrometers measurements. These simplifications will surely need to be reconsidered when this work is transposed to larger rockets.

\section{Trajectory estimation}

We now expose how the previously discussed model can be used to estimate the actual trajectory of the rocket from available measurements.

\section{A. A typical trajectory}

The trajectory of the rocket can be divided into four stages. The first one lasts until ignition. During this time period, the rocket is standing still on the launch-pad. It is possible to take advantage of this to calibrate the IMU sensors. During the second stage of the trajectory (Fig. 3a), the engine is on and the rocket is propelled and moving but remains on the launch ramp. The modeling of this period is quite uncertain due to unmodeled interactions with the ramp. Fortunately, the time spent moving on the ramp is very short.

Then, the third and main stage is the ascent flight of the rocket which is propelled. During this stage, the rocket accelerates before reaching its maximum velocity. Then, it slows down and reaches its peak altitude. Finally, the parachute is deployed at a time controlled by an onboard timer. Parachute deployment occurs 9 seconds after ignition. The predominant effect during this ascent stage is the thrust.

Finally, the fourth stage (Fig. 3b) is the descent flight when the rocket hangs under the parachute until its landing. During this period, the rocket and its parachute constitute a non-rigid flying object submitted to drag aerodynamics effects whose coefficients are badly known. ${ }^{9}$ Further, the system is quite sensitive to wind disturbances. For this reason, the fourth stage of the trajectory is not considered in the study. 


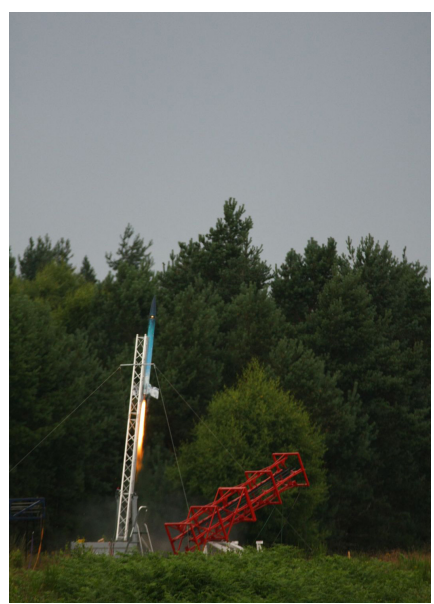

(a)

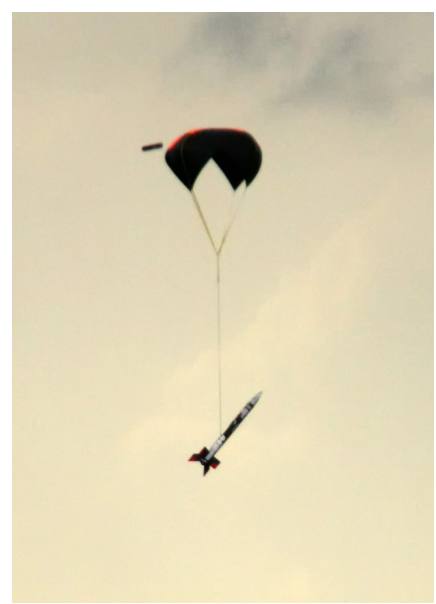

(b)

Figure 3. Rocket after ignition on the lauching ramp (a) and descent flight under parachute (b).

\section{B. Onboard instrumentation}

To reconstruct the ascent trajectory of the rocket, onboard measurements are available. Certainly, it would be possible and beneficial to consider ground instrumentations too, but so far this has been out of the scope of the experimentations.

Besides the flight management system (which manages mainly the opening of the parachute box thanks to the mentioned timer), the on-board flight system consists of two measurement sub-systems whose data are stored in a non-volatile memory. On one hand, there are the analog sensors for the engine. Their measurements are converted to digital data and transmitted to the on-board computer. On the other hand, the motion of the rocket is measured by an MTi $@$ IMU from XSens which delivers its measurements directly to the computer under the form of a periodic serial port message.

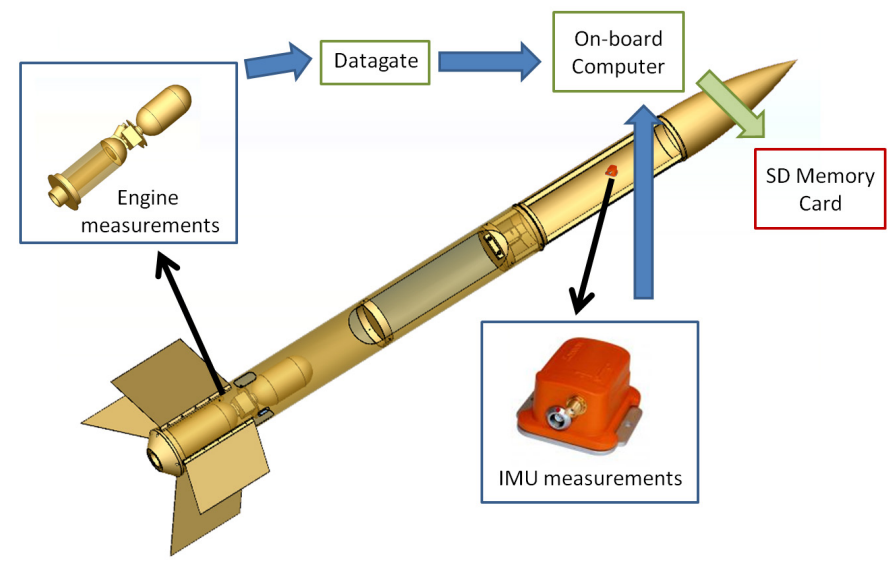

Figure 4. Measurement system.

\section{Inertial measurement unit}

The IMU delivers the acceleration vector (more precisely the acceleration minus gravity), the angular velocity vector and the magnetic field expressed in the body axes. For practical reasons, the IMU is not located at the center of gravity (Fig. 1,4). This complexifies the analysis as one must take into account lever arms which disturb the measurement of the accelerations by introducing angular velocities when they are not zero. The magnetic field measurements are heavily disturbed by the various metallic components of the rocket, and therefore left unused.

The IMU provides measurements at the frequency of $100 \mathrm{~Hz}$ with a bandwidth of $30 \mathrm{~Hz}$. The fast dynamics of the 
rocket can be reproduced well despite the saturation of the sensors $\left( \pm 300 \mathrm{deg} \cdot \mathrm{s}^{-1}\right.$ for gyrometers and $\pm 100 \mathrm{~m} \cdot \mathrm{s}^{-1}$ for accelerometers).

\section{Combustion system measurements}

The hybrid engine is equipped with a thermocouple along with a pressure transducer in the oxidizer tank and a pressure transducer in the combustion chamber. Each analog signal is sampled at $1 \mathrm{kHz}$. Interestingly, the high frequency of combustion measurements permits to take in account the phenomenon of combustion oscillations $(200 \mathrm{~Hz})$. These sensors ideally complement the IMU accelerometers which can only measure the low-pass content of the generated thrust. Finally, the initial amount of liquid oxidizer is evaluated from the weight of the whole system before and after filling of the tank. The burned mass of solid fuel is determined from the weight of the fuel grain at the beginning and at the end of the flight.

\section{Estimation technique}

Following the description of the rocket trajectory, its estimation is organized into independent steps. First, the thrust model is calibrated so that it is consistent with the mass conditions at initial and final times. Then, the IMU accelerometers measurements are used to compute a mean (fit) coefficient $\alpha$ between the theoretical thrust force $F_{T}$ obtained from the model and the effective thrust force $F_{t}$ exerted during the flight

$$
F_{t}=\alpha F_{T}
$$

The accelerometers are calibrated using data obtained when the rocket is on the launchpad. This allows to determine biases, scale factors, and misalignments.

As a second step, we use an extended Kalman filter $(\mathrm{EKF})^{10}$ during the ascent flight to reconciliate data from all the sensors. The filter runs with measured inputs (the thrust model) and measured outputs (the data from the IMU). The state vector gathers the state of the rocket considered as a 6 D-O-F rigid body (position, speed, attitude, angular velocity) and the rocket mass. To use the multirate signals from the sensors, the time step of the Kalman filtering prediction is $1 \mathrm{~ms}$ corresponding to the period of the measured inputs and the correction steps are synchronized to the IMU measurements and occur every $10 \mathrm{~ms}$.

\section{Trajectory estimation results}

Leaving out the parachute landing phase, which is heavily dependent on wind conditions, we focus on estimating the ascent trajectory of the rocket. Therefore, only the first three stages of the trajectory are considered. The goal here is to use state estimation to evaluate the efficiency of the hybrid engine and the culmination altitude.

In Fig. 5, we report estimates of the angular velocities, first the roll rate, then the pitch rate and finally the yaw rate. The roll rate shows an increase during the burn (which can be interpreted through the conservation of the kinetic momentum ${ }^{11}$ ) and then, it decreases slowly thanks to the stabilizing aerodynamic effect of the ailerons. On the transverse axis, the effects of the incidence oscillations appear. They are followed, 6 seconds later, by the slow rotation of the rocket towards the horizontal.

The estimates of the accelerations (see Fig. 5) illustrate the importance of the thrust model on the whole trajectory estimation. On the x-axis, the thrust dominates the dynamics. On the transverse axis, the residual misalignments (may be due to the centering of the engine or to the connection of the IMU to the frame of the rocket) appear. Later, they are followed by the (neglected) aerodynamics effects. At 8.5 seconds, the accelerometers detect the opening of the parachute box and one second later, the parachute leaves its pocket inside the rocket.

From the EKF estimation results, we also obtain the positions in an inertial frame centered on the launching pad. Fig. 7 reproduces the estimate of the three-dimensional trajectory. The initial pitch of 80 degrees leads to an ascent flight which is essentially vertical with a culmination altitude around 300 meters.

In more details, it is possible, through a parametric study, to evaluate the uncertainties in the trajectory reconstruction. Based on extensive numerical studies, it seems that, accounting for uncertainties in the determination of the exact trajectory timing (switch between the mentioned phases), and error in the fit of the combustion model, the main specifications of the trajectories can be evaluated as follows.

- Launch pad exit time $T_{E}=0.40 \pm 0.02 \mathrm{~s}$

- Launch pad exit speed $V_{E}=22.0 \pm 0.3 \mathrm{~m} \cdot \mathrm{s}^{-1}$ 

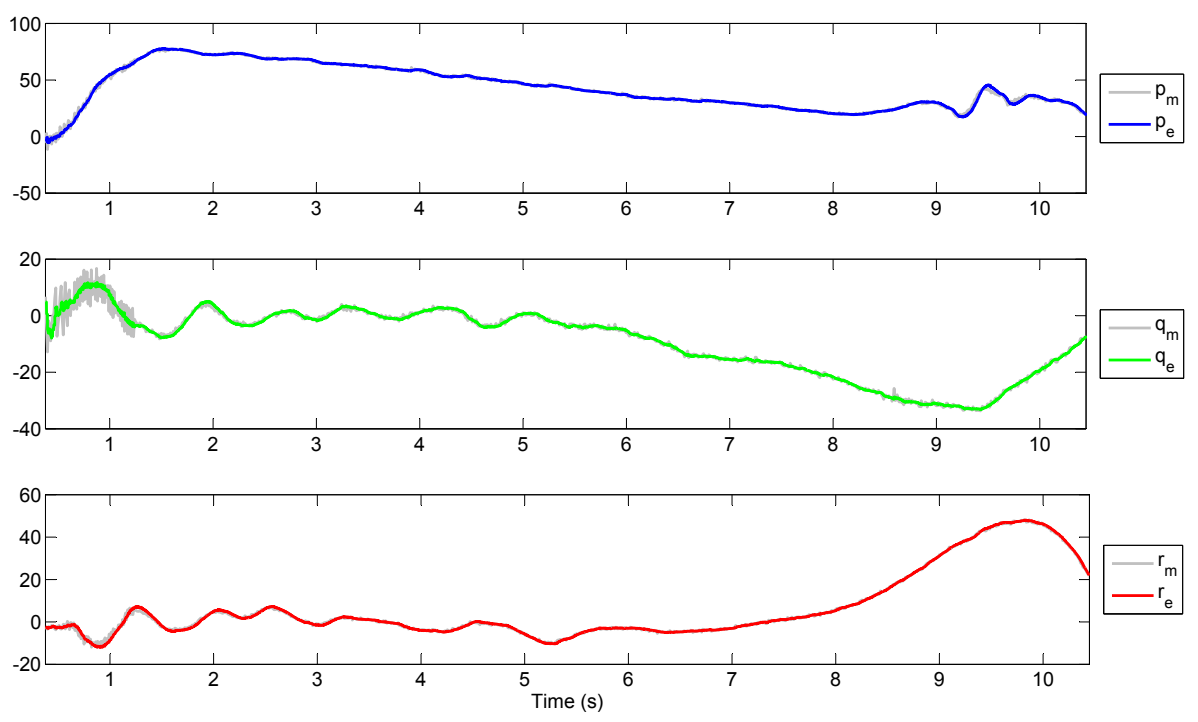

Figure 5. Estimated angular velocity (measurements in gray, estimation in color, deg.s ${ }^{-1}$ ). Roll, pitch and yaw rates.
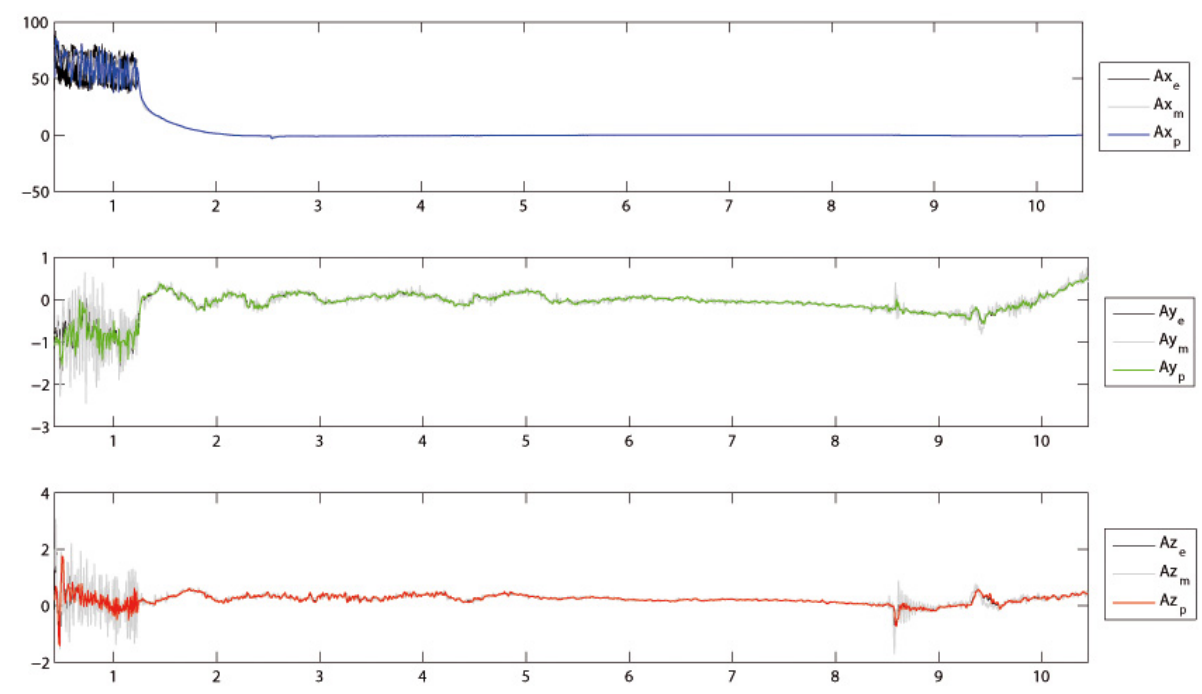

Figure 6. Estimated acceleration (measurements in gray, estimation in color, $\mathrm{m} . \mathrm{s}^{-2}$ ).

- Maximum speed $V_{\max }=65 \pm 1 \mathrm{~m} \cdot \mathrm{s}^{-1}$

- Culmination time $T_{C}=8.3 \pm 0.1 \mathrm{~s}$

- Culmination altitude $h_{C}=295 \pm 5 \mathrm{~m}$

Interestingly, the characteristic values of the hybrid engine can be computed from the results presented above.

\section{Conclusion}

As has been demonstrated on the case-study presented in this paper, trajectory estimation for a hybrid rocket based on low-cost sensors from the MEMS category can be achieved. This proves the relevance of the concept of data fusion of IMU measurements, engine combustion models, and measurements in this context. Certainly, the accuracy of the estimation can be improved by using additional sensors. These could be ground-based (e.g. theodolites, cameras) or located on-board (altimeter, or GPS for longer missions). Onboard camera can be also considered as a mean to evaluate the rotation of the rocket. To illustrate this point, the reader can refer to Fig. 8 where three successive snapshots from 


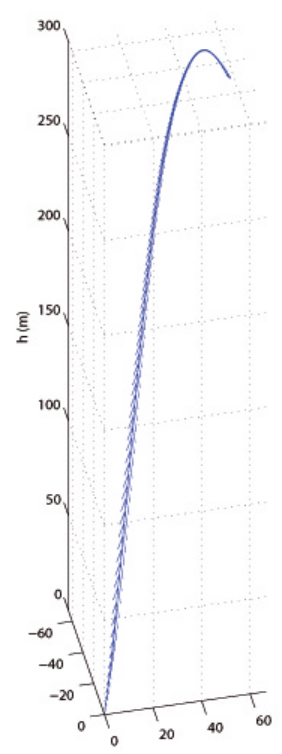

Figure 7. Estimated 3D trajectory $(\mathrm{m})$.

the onboard camera are given. The rocket rotation around the vertical axis is clearly visible. The work presented here represents only a first step toward a more general goal: trajectory estimation for (relatively) low-cost rockets using low-cost sensors. As a side product, the combustion model has been fitted using in-flight data. This is an interesting result since, despite its moderate accuracy compared to ground test-bench model identification ${ }^{1213}$, this approach is representative of in-flight engine behavior (which accounts for numerous disturbances such as aerodynamics effects, various oscillations, etc...). Future work should concentrate on the next generation of rockets in the PERSEUS program. Interestingly, hybrid rockets offer new control perspectives due to the possibility of throttling and restarting their engines. ${ }^{7}$ This potential will be investigated in the future.
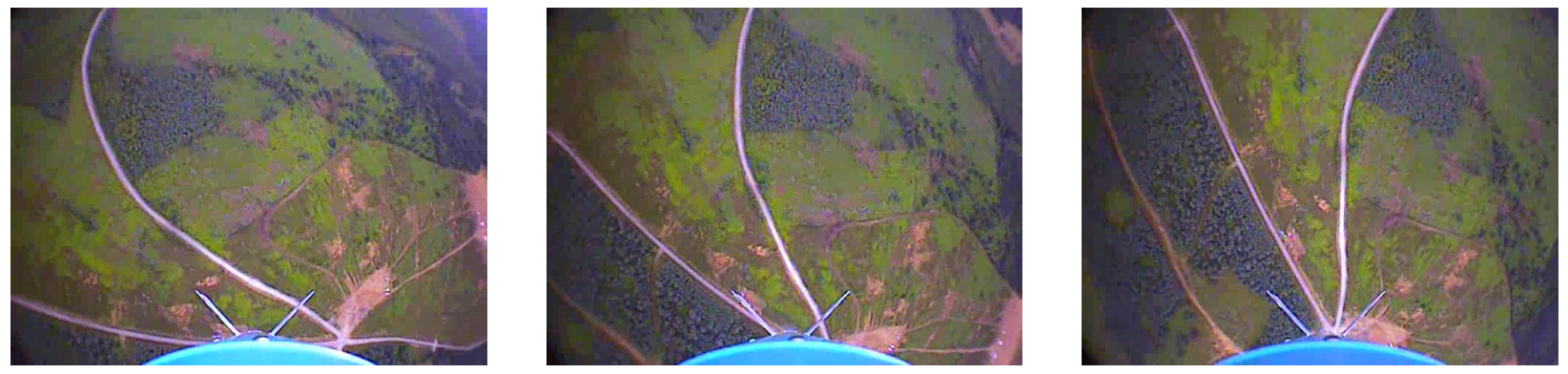

Figure 8. Potential use of the onboard camera as a rotation sensor.

\section{Acknowledgments}

The authors wish to thank F. Amouroux, B. Rmili, J. Franc, J. Oswald, L. Boireau, M. Prevost and the whole PERSEUS team for their scientific and technical support. This work was partially funded by CNES.

\section{References}

${ }^{1}$ Mukunda, H., Jain, V., and Paul, P., “A Review of Hybrid Rockets: Present Status and Future Potential,” Sadhana, May 1979.

${ }^{2}$ Nagata, H., Ito, M., Maeda, T., Watanabe, M., Uematsu, T., Totani, T., and Kudo, I., "Development of CAMUI Hybrid Rocket to Create a Market for Small Rocket Experiments," Acta Astronautica, Vol. 59, No. 1-5, 2006.

${ }^{3}$ Dupont, C., Bullock, M., Prevost, M., Maisonneuve, Y., Bec, R., Pillet, N., and Barenes, R., "The PERSEUS Student Launcher Project and Associated Hybrid Propulsion Activities," Proceedings of the 42th AIAA Joint Propulsion Conference and Exhibit, Sacramento, CA, July 2006. 
${ }^{4}$ Titterton, D. and Weston, J., Strapdown Inertial Navigation Technology, The American Institute of Aeronautics and Aeronautics, 2nd ed., 2004.

${ }^{5}$ Schulze, K. and Meyer, S., "The Rapid and Low-Cost Development of a Hybrid Rocket Motor," Proceedings of the 29th AIAA Joint Propulsion Conference and Exhibit, Monterey, CA, June 1993.

${ }^{6}$ Eke, F., "Dynamics of Variable Mass Systems," Tech. rep., Departement of Mechanical and Aeronautical Engineering, University of California, CA, 1999.

${ }^{7}$ Humble, R., Henry, G., and Larson, W., Space Propulsion Analysis and Design, The McGraw-Hill Companies Inc., 1995.

${ }^{8}$ Chiaverini, M. and Kuo, K., editors, Fundamentals of Hybrid Rocket Combustion and Propulsion, American Institute of Aeronautics and Astronautics, Inc., 2007.

${ }^{9}$ Dobrokhodov, V., Yakimenko, O., and C.J.Junge, "Six-Degree-Of-Freedom Model of a Controlled Circular Parachute," Journal of Aircraft, Vol. 40, No. 3, June 2003.

${ }^{10}$ Stengel, R., Optimal Control and Estimation, General Publishing Company, Ltd., 2nd ed., 1994.

${ }^{11}$ Eke, F., Mao, T.-C., and Morris, M., "Free Attitude Motions of a Spinning Body with Substantial Mass Loss," Journal of Applied Mechanics, Vol. 71, No. 2, March 2004.

${ }^{12}$ Lohner, K., Dyer, J., Doran, E., Dunn, Z., and Zilliac, G., "Fuel Regression Rate Characterization Using a Laboratory Scale Nitrous Oxide Hybrid Propulsion System," Proceedings of the 42th AIAA Joint Propulsion Conference and Exhibit, Sacramento, CA, July 2006.

${ }^{13}$ Stamatov, V., Honnery, D., and Soria, J., "Visualization of Flow Development in Hybrid Rocket Motors with High Regression Rates," Journal of Propulsion and Power, Vol. 21, No. 4, 2005. 